\title{
Characterization of Dominance Relations in Finite Coalitional Games
}

\author{
Felix Brandt Paul Harrenstein \\ Ludwig-Maximilians-Universität München, Germany \\ \{brandtf, harrenst\}@tcs.ifi.lmu.de
}

\begin{abstract}
McGarvey (1953) has shown that any irreflexive and anti-symmetric relation can be obtained as a relation induced by majority rule. We address the analogous issue for dominance relations of finite cooperative games with non-transferable utility (coalitional NTU games). We find any irreflexive relation over a finite set can be obtained as the dominance relation of some finite coalitional NTU game. We also show that any such dominance relation is induced by a non-cooperative game through $\beta$-effectivity. Dominance relations obtainable through $\alpha$-effectivity, however, have to comply with a more restrictive condition, which we refer to as the edge-mapping property.
\end{abstract}

Keywords: Cooperative Game Theory, Non-Transferable Utility, Dominance

\section{Introduction}

Many important concepts in the mathematical social sciences are defined in terms of a binary dominance relation on a set of outcomes or alternatives. These concepts can be applied to any model of social interaction for which such a concept of dominance can be meaningfully defined. For example, the set of undominated outcomes defines Gillies' core in cooperative game theory, Nash's solution in the bargaining problem, or, more generally, the idea of Pareto optimality (see Aumann, 1961, p. 539). Other notions that are similarly defined in terms of dominance are von Neumann-Morgenstern stable sets for cooperative games as well as the Condorcet winner along with Condorcet consistent choice rules, such as the Banks set, the uncovered set, and Dutta's minimal covering set in social choice theory (see, e.g., Laslier, 1997). ${ }^{1}$

Different models of interaction call for different notions of dominance. In social choice theory, for example, dominance is defined with respect to a profile of individual preferences over a set of social alternatives. Although other definitions are also possible, typically, an alternative $a$ is then said to dominate another alternative $b$ if the number of individuals preferring $a$ to $b$ exceeds the number of individuals preferring $b$ to $a$. In coalitional games, on the other hand, the concept of dominance is generally defined in terms of coalitional effectivity and individual preferences. Effectivity can be defined in a number of ways, each of which reflects the power of coalitions in terms of the outcomes they can enforce to come about. An outcome $a$ is then said to dominate another outcome $b$ if some coalition is effective for $a$ and, moreover, all members of 
that coalition prefer outcome $a$ to outcome $b$. Thus, cooperative majority voting can be seen as the special case in which majorities are effective for any outcome (see also Taylor and Zwicker, 1999).

In either case, the dominance relation need not generally be transitive and may even contain cycles. Accordingly, the common concept of maximality is no longer tenable with respect to the dominance relation and new concepts have been developed to take over its function of singling out elements that are in some sense primary. Von Neumann and Morgenstern considered this phenomenon as one of the most fundamental problems the mathematical social sciences have to cope with (see von Neumann and Morgenstern, 1947, Chapter 1). On this account, each of the concepts mentioned above, be their roots in social choice theory or in cooperative game theory, has to deal with what is essentially the same problem: to come to grips with a possibly intransitive dominance relation. Each of them incorporates a different intuition and approaches the issue from a different angle.

The dominance relations themselves, however, have different structural properties in both disciplines. As it is defined for social choice on the basis of majority rule, the dominance relation is asymmetric, i.e., both irreflexive and anti-symmetric. In coalitional games the dominance relation is also irreflexive, but not generally anti-symmetric. The structural properties of a dominance-based solution concept, such as existence and uniqueness, may vary, depending on properties of the underlying dominance relation. Therefore, to judge the merits of a particular dominance-based solution concept as a substitute for maximality, one need to know the structural properties of the dominance relation. In this vein, McGarvey (1953) has shown that, in the setting of majority voting, it is precisely the asymmetric relations on a finite set of alternatives that can be obtained as the dominance relation for some profile of linear preferences over those alternatives.

We take up the analogous issue for finite coalitional games with non-transferable utility or finite coalitional NTU games and give complete characterizations of the structural properties of the dominance relations for three classes of such games.

There is a range of environments that are best modeled by assuming an infinite number of alternatives (see, e.g., Bergin and Duggan, 1999). For instance, if coalitions are in a position to play correlated mixed strategies, the outcomes of a coalitional game are a convex and compact subset of Euclidean space. In other contexts it is more natural, desirable, or convenient to consider a finite number of outcomes. This is, e.g., the case in situations where mixed strategies are suspect or unnatural. In matters of life and death players and coalitions may not be willing to have their behavior depend on some randomization device. In other contexts, mixed strategies may simply not be available (see, e.g., Luce and Raiffa, 1957, Section 4.10 for an early discussion). A finite number of outcomes is also a common and simplifying assumption in bilateral bargaining (Kıbris and Sertel, 2007) and cooperative majority voting games, which, interestingly, is exactly where social choice and cooperative game theory intersect (see, e.g., Schwartz, 1990; Dutta and Laslier, 1999). Our results pertain to NTU games with a finite number of outcomes (see, e.g., Farquharson, 1969; Kalai and Samet, 1985; Abdou and Keiding, 1991; Lahiri, 2007).

Another noteworthy property of the coalitional model studied in this paper is the way the utility a coalition can guarantee its members is related to actual outcomes. In particular, our finite model does not assume comprehensiveness of the coalitional effectivity functions, in the 


\begin{tabular}{lll}
\hline Dominance Relation & Properties & Result \\
\hline Finite NTU games & irreflexivity & Theorem 1 \\
Finite NTU games through $\beta$-effectivity & irreflexivity & Theorem 2 \\
Finite NTU games through $\alpha$-effectivity & irreflexivity and EMP & Theorem 3 \\
\hline
\end{tabular}

Table 1: Characterizing properties of the various types of dominance relation. The edgemapping property $(E M P)$ is defined in Section 4.

sense that if a coalition can guarantee its members a particular utility, it can also guarantee them any lesser utility. Here, we take a more general approach. We assume every finite NTU game to be subject to a so-called comprehension condition, which, as a function of the finite set of outcomes, determines the range of utility vectors the various coalitions can be feasible for. Thus, comprehensiveness can be accounted for by imposing a very liberal comprehension condition. On the other extreme, the comprehension condition can be tight, meaning that coalitions can only be effective for utility vectors that are actually instantiated by one of the outcomes. How natural the assumptions of comprehensiveness and tightness are largely depends on the setting that is being considered (see Bergin and Duggan, 1999, for an interesting discussion of comprehensiveness). Our results hold for every comprehension condition and are thus independent of any specific choice in this respect.

Our first result pertains to the dominance relations of general finite NTU games. We find that every irreflexive relation on a finite set $A$ of alternatives can be obtained as the dominance relation of some finite coalitional NTU game. Coalitional NTU games can also be obtained from non-cooperative games, in particular normal-form games, in a variety of ways. Traditionally, the notions of $\alpha$ - and $\beta$-effectivity are employed to obtain the characteristic function of a coalitional NTU game (Aumann, 1959; Aumann and Peleg, 1960). It turns out that the structural properties of the dominance relations of finite coalitional NTU games obtained through $\beta$-effectivity are identical to those of the general case. The dominance relation induced by a finite NTU game obtained through $\beta$-effectivity may be any irreflexive relation. The formal properties of dominance relations obtained through $\alpha$-effectivity, however, are subject to narrower constraints. We find that they are characterized by irreflexivity and the edge-mapping property, a structural property defined in this paper. Table 1 summarizes our results.

The significance of these results is mainly of theoretical nature. They show which structural properties of the dominance relation one can rely on when proving something about a dominance-based concept in finite NTU games. On the other hand, they also determine the extent of freedom one has in constructing counterexamples. In this context, it is also worth mentioning that McGarvey's characterization result was motivated by the construction of voting paradoxes (McGarvey, 1953).

There is also an interesting conceptual connection between this paper and the literature on non-cooperative foundations of cooperative solutions, also commonly referred to as the Nash program. This line of research aims to provide non-cooperative models, e.g., bargaining environments (Nash, 1950, 1953), in which the cooperative and non-cooperative solutions coincide (e.g., Rubinstein, 1982; Hart and MasColell, 1996). Another connection concerns the theory of 
implementation (e.g., Serrano, 1997; Bergin and Duggan, 1999). Assuming comprehensiveness, Bergin and Duggan (1999) completely characterized the coalitional games that can be obtained from strategic environments both through $\alpha$ - and $\beta$-effectivity. The objective of this paper, however, is different, as it aims to fully characterize the structural properties of the cooperative games obtained in this manner, rather than the games themselves. ${ }^{2}$

\section{Finite Coalitional NTU Games}

The intuition underlying the models of coalitional game theory is that the players can make binding commitments, form coalitions, and thus correlate their actions. Here we consider the general case without the possibility of side-payments, i.e., we do not hypothesize the existence of a transferable commodity with which all players' preferences are positively associated. We do, however, assume the set of possible outcomes to be finite.

Formally, in our framework we consider finite populations $N=\{1, \ldots,|N|\}$ of individuals or players and finite sets $A=\left\{a_{1}, \ldots, a_{|A|}\right\}$ of outcomes or alternatives. A coalition $C$ is a nonempty subset of $N$ and we have $-C$ denote the complement $N \backslash C$ of $C$ in $N$. The players entertain preferences over $A$, which we assume to be represented by a real-valued $|N| \times|A|$ utility matrix $U=\left(u_{i j}\right)_{i \in N, j \in A}$, where $u_{i j}$ denotes the utility of the $j$ th outcome to the $i$ th player. Thus, each row $\left(u_{i 1}, \ldots, u_{i|A|}\right)$ could be construed as representing player $i$ 's utility function over $A$. Accordingly we also write $u_{i}\left(a_{j}\right)$ for $u_{i j}$ and $u_{i}$ for the entire row. Similarly, for each coalition $C$ we have $u_{C}(a)$ denote $\left(u_{i}(a)\right)_{i \in C}$. On the other hand, each column $\left(u_{1 a}, \ldots, u_{|N| a}\right)$ is a utility vector in $\mathbb{R}^{N}$, which we write as $u(a)$. Given a utility matrix $U$, we have $H(U)$ denote the set $\{u(a): a \in A\}$ of feasible utility vectors in $U$, omitting the reference to $U$ when it is fixed in the context.

For a coalition $C \subseteq N$ and a $x=\left(x_{i}\right)_{i \in N}$ in $\mathbb{R}^{N}$ we have $x_{C}$ denote the vector $\left(x_{i}\right)_{i \in C}$. For $X \subseteq$ $\mathbb{R}^{N}$ we also write $X_{C}=\left\{x_{C}: x \in X\right\}$. With a slight abuse of notation, we write $x_{\left\{i_{1}, \ldots, i_{k}\right\}}$ for $\left(x_{i_{1}}, \ldots, x_{i_{k}}\right)$, assuming the order of the players to be fixed. If $C$ and $D$ are disjoint coalitions and $x_{C} \in \mathbb{R}^{C}$ and $y_{D} \in \mathbb{R}^{D}$, let $\left(x_{C}, y_{D}\right)$ denote the utility vector $\left(z_{i}\right)_{i \in C \cup D} \in \mathbb{R}^{C \cup D}$ with $z_{i}=x_{i}$, if $i \in C$, and $z_{i}=y_{i}$, if $i \in D$. We also write $x_{C} \geq y_{C}$ in case $x_{i} \geq y_{i}$ for all $i \in C$ and $x_{C}>y_{C}$ if $x_{i}>y_{i}$ for all $i \in C$.

By a comprehension condition we understand a function $\chi$ that associates each subset $X \subseteq \mathbb{R}^{n}$ with a superset $\chi(X) \subseteq \mathbb{R}^{n}$ of $X$. We assume comprehension conditions to be downward, i.e., for all $X \subseteq \mathbb{R}^{n}, X \subseteq \chi(X) \subseteq \bigcup_{x \in X}\left\{y \in \mathbb{R}^{n}: x \geq y\right\}$. The largest comprehension condition, i.e., the one with $\chi(X)=\bigcup_{x \in X}\left\{y \in \mathbb{R}^{n}: x \geq y\right\}$ for all $X$, we call (full) comprehensiveness, whereas the smallest, i.e., the one for which $\chi(X)=X$ for all $X \subseteq \mathbb{R}^{N}$, we refer to as tight. It is worth observing that the above assumption excludes the convex hull as a comprehension condition.

Given comprehension condition $\chi$, a characteristic function $V$ under $\chi$ with co-domain $X \subseteq$ $\mathbb{R}^{N}$ maps each coalition $C \subseteq N$ to a non-empty subset $V(C)$ of utility vectors in $\chi\left(X_{C}\right)$. We usually omit the subscript if the comprehension condition is implicit in the context. Intuitively, a characteristic function associates with each coalition a set of utility vectors the coalition can guarantee its members. What this guarantee amounts to is left implicit.

Definition 1 (Finite NTU game). Given a comprehension condition $\chi$, a finite coalitional game with non-transferable utility or finite NTU game is a tuple $\left(N, H, V_{\chi}\right)$, where $N$ is set of players, 
$H$ is a finite subset of $\mathbb{R}^{N}$, and $V_{\chi}$ is a characteristic function under $\chi$ with co-domain $H$.

A finite NTU game $(N, H, V)$ is comprehensive if for each coalition $C, y_{C} \in \mathbb{R}^{C}$ and $x_{C} \in$ $V(C), x_{C} \geq y_{C}$ implies $y_{C} \in V(C)$. Similarly, we say $(N, H, V)$ is tight if the associated comprehension condition is tight and, consequently, $V(C) \subseteq H_{C}$, for all coalitions $C$. A finite NTU game is said to be ordinary whenever $H \subseteq V(N)$, i.e., if the grand coalition $N$ of all players is effective for every feasible outcome (see, e.g., Aumann, 1961). It is monotonic in case $C \subseteq D$ and $x_{C} \in V(C)$ imply that there is some $y \in V(D)$ such that $y_{C} \geq x_{C}$, i.e., if a coalition can guarantee its members at least as much as each of its subcoalitions. A stronger condition is that of superadditivity, which a characteristic function $V$ satisfies if for all disjoint coalitions $C$ and $D$, $x_{C} \in V(C)$ and $y_{D} \in V(D)$ imply $\left(x_{C}, y_{D}\right) \in V(C \cup D)$. Superadditivity implies monotonicity but not vice versa. Finally, a finite NTU game is binary if $H \subseteq\{0,1\}^{N}$.

We generally assume the set of feasible utility vectors of a finite NTU $(N, H, V)$ game to be given by the utility vectors of a utility matrix over a finite set of outcomes, i.e., $H=H(U)$ for some utility matrix $U$ over $A$. Each utility vector in $H$, thus, represents a distribution of utility that can actually come about. On the other hand, the utility vectors in $V(C)$ for which a coalition $C$ is effective and which need not all be included in $H_{C}$ could be interpreted as representing the bargaining position of $C$. The comprehension condition determines how the bargaining position is related to the outcomes the coalition can achieve. More particularly, a coalition's bargaining position may be based on the sets of outcomes within which it can enforce the outcome to fall, rather than particular individual outcomes it can force to come about. Thus, a coalition $C$ may be able to guarantee that the outcome is among $a$ and $b$ but cannot enforce either $a$ or $b$ separately. Suppose that $C$ consists of two players, 1 and 2, and the utility vectors associated with $a$ and $b$ for $C$ are given by $u(a)=(2,1)$ and $u(b)=(1,2)$. The coalition $C$ could then demand a utility of 1 to both of its members on this basis, even if there is no outcome that yields precisely 1 to both player 1 and player 2 . If the circumstances are such that such a demand can reasonably be made, the utility vector $(1,1)$ should be included in $V(C)$, calling for a comprehension condition that makes this possible. On the other hand, if no such claim can be made, coalition $C$ should not be effective for $(1,1)$ and the situation should be modeled by means of a tighter comprehension condition.

Formally, a utility vector $u$ in $\mathbb{R}^{N}$ is said to be feasible for $C$ if there is some $x_{C} \in V(C)$ with $x_{C} \geq u_{C}$. We also say that a coalition is effective for a utility vector $u$ if $u$ is feasible for $C$. We also say that a coalition is effective for an outcome $a$ if $C$ is effective for $x_{C}$ and $x_{C}=u_{C}(a)$ for some outcome $a$. Now, the notion of dominance in NTU games is defined in terms of players' preferences and coalitional effectivity.

Definition 2 (Dominance). Let $(N, H, V)$ be a finite NTU game and let $C$ be a coalition. For $u$ and $u^{\prime}$ utility vectors in $H$, we say $u$ dominates $u^{\prime}$ via $C$, in symbols $u>_{C} u^{\prime}$, if $u$ is feasible for $C$ and $u_{C}>u_{C}^{\prime}$, i.e., if there is some $x_{C} \in V(C)$ with $x_{C} \geq u_{C}>u_{C}^{\prime}$. Utility vector $u$ dominates $u^{\prime}$, in symbols $u>u^{\prime}$, whenever $u$ dominates $u^{\prime}$ via some coalition $C$.

Obviously no utility vector dominates itself, i.e., the dominance relation for NTU games is irreflexive.

Let $H$ be a set of feasible utility vectors defined by a utility matrix $U$ which defines the players' preferences over a finite set of outcomes $A$. Then, every dominance relation on $H$ 
straightforwardly defines a dominance relation on $A$. Thus, we say that outcome a dominates outcome $b$ whenever $u(a)$ dominates $u(b)$. More formally, we say that a binary relation $R$ on a finite set $A=\left\{a_{1}, \ldots, a_{|A|}\right\}$ is induced by a finite NTU game $(N, H, V)$ whenever a utility matrix $U=\left(u_{i j}\right)_{i \in N, j \in A}$ exists such that $H=H(U),|H|=|A|$ and, for all $a, b \in A, a R b$ if and only if $a$ dominates $b$, i.e., if the function which maps each $a \in A$ to the vector $u(a) \in H$ is an isomorphism between the graphs $(A, R)$ and $(H,>)$. We now have the following useful lemma, which basically says that, for our purposes and as far as the structure of the dominance relations is concerned, we can restrict our attention to tight games without loss of generality.

Lemma 1. Let $(N, H, V)$ be a tight finite $N T U$ game and $\chi$ a comprehension condition. Then, there is a finite $N T U$ game $\left(N, H, V^{\prime}\right)$ under $\chi$ such that the dominance relations of $(N, H, V)$ and $\left(N, H, V^{\prime}\right)$ coincide.

Proof. Define the characteristic function $V^{\prime}$ such that $V^{\prime}(C)=\chi(V(C))$ for every coalition $C \subseteq$ $N$. Consider the finite NTU game $\left(N, H, V^{\prime}\right)$ and let $>$ and $>^{\prime}$ denote the dominance relations of $(N, H, V)$ and $\left(N, H, V^{\prime}\right)$, respectively. We show that $u>u^{\prime}$ if and only if $u>^{\prime} u^{\prime}$ for all $u, u^{\prime} \in$ $H$. First assume $u>u^{\prime}$. Then there is some coalition $C$ and some $x_{C} \in V(C)$ such that $x_{C} \geq$ $u_{C}>u_{C}^{\prime}$. Hence, $x_{C} \in \chi(V(C))$ and, accordingly, $x_{C} \in V^{\prime}(C)$. Therefore also $u>^{\prime} u^{\prime}$. For the other direction, assume $u>^{\prime} u^{\prime}$. Then there is some coalition $C$ and some $x_{C} \in V^{\prime}(C)$ such that $x_{C} \geq u_{C}>u_{C}^{\prime}$. As $x_{C} \in \chi(V(C))$ and comprehension conditions are downward, there is some $y_{C} \in V(C)$ such that $y_{C} \geq x_{C}$. It follows that $y_{C} \geq u_{C}>u_{C}^{\prime}$. Hence, $u>u^{\prime}$.

\section{Dominance Relations of Finite NTU Games}

We are now in a position to prove our first result, which states that every irreflexive relation on a finite set of outcomes can be induced as the dominance relation of some finite NTU game. The idea behind the proof is to construct a coalitional game for each irreflexive relation $R$ on a set of outcomes $A$. We introduce two players $i_{a}$ and $j_{a}$ for each $a \in A$ as well as an appropriate utility matrix $U$, which depends on $R$ and represents the players' preferences over $A$. The set $H$ feasible utility vectors is then given by $H(U)=\{u(a): a \in A\}$. Each coalition that contains both $i_{a}$ and $j_{a}$ for some $a \in A$, is defined to be universally effective, i.e., any such coalition $C$ feasible for any vector in $H_{C}$, whereas any other coalition $D$ is so ineffective that no vector in $H$ dominates any other via $D$. The reader is referred to Figure 1 for an illustration of this construction, which will also be used in the proof of Theorem 2 below. In Theorem 1 we formally define for each irreflexive relation $R$ a collection of finite NTU games and establish that the dominance relation on $A$ as induced by each of these games coincides with $R$.

Theorem 1. Let $R$ be an irreflexive relation on a finite set $A$ of outcomes and $\chi$ a comprehension condition. Then, $R$ is induced as the dominance relation of some finite NTU game under $\chi$.

Proof. By virtue of Lemma 1, we may that $\chi$ is tight. For $R$ we define a collection of finite NTU games $V_{R}=(N, H, V)$, which only differ with respect to their characteristic functions.

With each $a \in A$ we associate two players $i_{a}$ and $j_{a}$, and say that $\left\{i_{a}, j_{a}\right\}$ are $a$ pair and that $i_{a}$ and $j_{a}$ are partners. Formally, $N=\{1, \ldots, 2|A|\}$ and let $\left\{X_{1}, \ldots, X_{|A|}\right\}$ be a partitioning of $N$ 


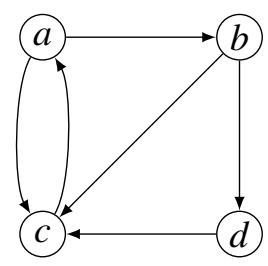

$\begin{array}{cccccccc}i_{a} & j_{a} & i_{b} & j_{b} & i_{c} & j_{c} & i_{d} & j_{d} \\ a \\ b \\ c \\ d\end{array}$
$\left.\begin{array}{llllllllll}1 & 1 & 0 & 1 & 0 & 0 & 0 & 1 \\ 0 & 0 & 1 & 1 & 0 & 1 & 0 & 1 \\ 0 & 0 & 0 & 0 & 1 & 1 & 0 & 0 \\ 0 & 1 & 0 & 0 & 0 & 1 & 1 & 1\end{array}\right)$

Figure 1: The graph of an irreflexive relation that is induced by a finite NTU game $(N, H, V)$ where $N$ is given by $\left\{i_{a}, j_{a}, i_{b}, j_{b}, i_{c}, j_{c}, i_{d}, j_{d}\right\}$. The players' preferences are given by the utility matrix $U$. For reasons of readability we depict the transposal $U^{\top}$ of $U$ on the right.

with $\left|X_{k}\right|=2$ for all $1 \leq k \leq|A|$. We associate each $X_{k}$ with outcome $a_{k}$ and write $X_{k}=\left\{i_{a_{k}}, j_{a_{k}}\right\}$. Let $U=\left(u_{i j}\right)_{i \in N, j \in\{1, \ldots,|A|\}}$ be the $|N| \times|A|$ utility matrix such that for all $a, b \in A$,

$$
u_{\left\{i_{a}, j_{a}\right\}}(b)= \begin{cases}(1,1) & \text { if } a=b, \\ (0,0) & \text { if } a \neq b \text { and } a R b, \\ (0,1) & \text { otherwise. }\end{cases}
$$

Set $H=H(U)=\{u(a): a \in A\}$. Observe that $u(a)=u(b)$ if and only if $a=b$. Hence, $|A|=|H|$.

Let for each coalition $C, H_{C}^{*}$ denote the subset of utility vectors in $H_{C}$ that minimize the utility of at least one of $C$ 's members, i.e.,

$$
H_{C}^{*}=\bigcup_{i \in C}\left\{x_{C} \in H_{C}: x_{i}=\min _{u \in H} u_{i}\right\}
$$

As $H$ is assumed to be finite, this set is well defined.

A characteristic function $V$ for a game in the collection for $R$ is then defined such that for each coalition $C$ in $N$,

$$
V(C)= \begin{cases}H_{C} & \text { if }\left\{i_{a}, j_{a}\right\} \subseteq C, \text { for some } a \in A, \\ X_{C}^{*} & \text { otherwise }\end{cases}
$$

for some $X_{C}^{*} \subseteq H_{C}^{*}$. We show for every game in the collection of games for $R$ that for all $a, b \in A$ we have $a R b$ if and only if $a$ dominates $b$.

First assume that $a R b$. Observe that, by construction, $u_{\left\{i_{a}, j_{a}\right\}}(a)=(1,1)$ and $u_{\left\{i_{a}, j_{a}\right\}}(b)=$ $(0,0)$. Moreover, $(1,1) \in V\left(\left\{i_{a}, j_{a}\right\}\right)$. Accordingly, $u(a)$ dominates $u(b)$ via the coalition $\left\{i_{a}, j_{a}\right\}$ and we may conclude that $a$ dominates $b$ in $V_{R}$.

For the opposite direction, assume that $a$ dominates $b$ in $V_{R}$. Then, there is some coalition $C$ and some $x_{C} \in V(C)$ such that $x_{C} \geq u_{C}(a)>u_{C}(b)$. Because $V_{R}$ is binary, for each $i \in C$ we have $x_{i}=u_{i}(a)=1$ and $u_{i}(b)=0$. Accordingly, $\min _{u \in H} u_{i}=0$ for each $i \in C$. As $x_{C} \in V(C)$, there is some $c \in A$ with $\left\{i_{c}, j_{c}\right\} \subseteq C$ and $u_{\left\{i_{c}, j_{c}\right\}}(a)=(1,1)$. By definition of $B$, then, $a=c$. Hence, $j_{a} \in C$ and $u_{j_{a}}(b)=0$ and, by construction, it follows that $a R b$.

It is readily appreciated that the finite NTU game $V_{R}$ constructed in the proof above is binary, ordinary, and monotonic. Moreover, the construction used in the proof of Lemma 1 can easily 
be seen to preserve these properties. Accordingly, under every comprehension condition there is some finite NTU game that corresponds to some irreflexive binary relation and, moreover, satisfies these natural properties.

\section{Coalitional Effectivity in Non-Cooperative Games}

Although in the formal definition of a finite NTU game the players' strategies are abstracted away, they are still implicit in the characteristic function. A coalition is assumed to be effective for a particular utility vector if its members have a joint strategy that guarantees all of them the utility specified in that vector. However, the definition gives no formal and precise interpretation of this guarantee. The question keeps lingering how this guarantee should be given a formal and precise interpretation. In a setting without transferable utility, $\alpha$ - and $\beta$-effectivity provide two standard ways of determining the value of a coalition in a non-cooperative game (see, e.g., Aumann, 1959, 1961; Aumann and Peleg, 1960). After having introduced the appropriate formal definitions of a non-cooperative game in normal form and those of $\alpha$-effectivity and $\beta$-effectivity, we show that irreflexivity characterizes dominance relations of NTU games obtained through $\beta$ effectivity. Dominance relations of NTU games obtained through $\alpha$-effectivity, however, are subject to more restrictive constraints.

Definition 3 (Normal-form game). A game $G$ in normal form is a tuple $(N, S, \Omega, g, U)$, where $N$ is a set $\{1, \ldots,|N|\}$ of players, $S=\chi_{i \in N} S_{i}$ is an $|N|$-dimensional space of strategy profiles, $\Omega=\left\{\omega_{1}, \ldots, \omega_{|\Omega|}\right\}$ a set of outcomes, and $g: S \rightarrow \Omega$ an outcome function associating each strategy profile $s$ with an outcome $g(s)$ in $\Omega$. Finally, $U=\left(u_{i j}\right)_{i \in N, j \in \Omega}$ is a real-valued $|N| \times|\Omega|$ utility matrix.

We have $\left(s_{C}, t_{-C}\right)$ denote the strategy profile $s^{*}$ such that $s_{i}^{*}=s_{i}$ if $i \in C$ and $s_{i}^{*}=t_{i}$ if $i \notin C$.

A coalition $C$ is said to be $\alpha$-effective for a particular utility vector $x_{C} \in \mathbb{R}^{C}$, if in the normalform game coalition $C$ has a joint strategy that guarantees each of its members $i$ at least a utility of $x_{i}$, no matter which strategies the players not in $C$ may adopt. By contrast, $C$ is said to be $\beta$-effective for a particular utility vector $x_{C} \in \mathbb{R}^{C}$, if the players that are not in $C$ have no joint strategy that precludes the coalition $C$ from obtaining a utility of at least $x_{i}$ to each of its members $i$.

Definition 4 ( $\alpha$-effectivity and $\beta$-effectivity). Let $G=(N, S, \Omega, g, U)$ be a game in normal form, $C$ a coalition in $N$ and $x_{C} \in \mathbb{R}^{C}$. Then,

$C$ is $\alpha$-effective for $x_{C}$ if there is an $s \in S$ such that for all $t \in S, u_{C}\left(g\left(s_{C}, t_{-C}\right)\right) \geq x_{C}$,

$C$ is $\beta$-effective for $x_{C}$ if for all $s \in S$, there is a $t \in S$ such that $u_{C}\left(g\left(t_{C}, s_{-C}\right)\right) \geq x_{C}$.

For $\gamma \in\{\alpha, \beta\}$ and $\chi$ a comprehension condition, a finite NTU game $(N, H, V)$ is said to $\gamma$ correspond to a normal-form game $G=(N, S, \Omega, g, U)$ under $\chi$, whenever $H=\{u(g(s)): s \in S\}$ and for each coalition $C$ in $N$,

$$
V(C)=\left\{x_{C} \in \chi\left(H_{C}\right): C \text { is } \gamma \text {-effective for } x_{C}\right\}
$$


Also, if a binary relation $R$ on a set $A$ can be induced as the dominance relation of some finite NTU game under $\chi$ that $\gamma$-corresponds to some normal-form game, we say that $R$ is obtainable through $\gamma$-effectivity under $\chi$. If the comprehension condition $\chi$ is clear from the context, we usually omit the reference to $\chi$.

The following example concerns a class of normal-form games that evince a particularly sharp contrast between the sets of outcomes for which coalitions are $\alpha$-and $\beta$-effective.

Example 1. Let $\Omega=\left\{\omega_{1}, \ldots, \omega_{|\Omega|}\right\}$ be a set of outcomes, $N$ a set of players, and $k \in \mathbb{N}$. Let further $\phi: \mathbb{N}^{N} \rightarrow \mathbb{N}$ such that for each $x \in \mathbb{N}^{N}$,

$$
\phi(x)=1+\left(\sum_{i \in N} x_{i} \bmod |\Omega|\right) .
$$

The modulo game $M(\Omega, k)=(N, S, \Omega, g, U)$ on $\Omega$ and $k$ is then a game in normal form such that for each player $i, S_{i}=\{1, \ldots, k\}$ and $g$ such that for each strategy profile $s \in S, g(s)=a_{k}$ if and only if $k=\phi(s)$. Obviously, if $k \geq|\Omega|$, for every $\omega_{m} \in \Omega$ and every joint strategy $s_{-C}$ of its non-members, every coalition $C$ has a strategy $t_{C}$ that yields $a_{m}$ as the outcome of the modulo game $M(\Omega, k)$. Merely set $t_{C}$ such that $\left.m=1+\left(\left(\sum_{i \in C} t_{i}+\sum_{i \notin C} s_{i}\right) \bmod |\Omega|\right)\right)$. As this is always possible, every coalition is $\beta$-effective for every outcome in $\Omega$, whereas every coalition other than the grand coalition $N$ is only $\alpha$-effective for outcomes in $\Omega$ that minimize the utility of at least one of its members.

As for the general case, we find that the structure of dominance relations induced by finite NTU games do not depend on the comprehension condition assumed. Rather, in order to establish the characterizing structural properties of the dominance relations obtained through either $\alpha$ - or $\beta$-effectivity, we can assume the comprehension condition to be tight.

Lemma 2. Let $\gamma \in\{\alpha, \beta\}, R$ be a binary relation on a finite set $A$, and $\chi$ a comprehension condition. Then, if $R$ is obtainable trough $\gamma$-effectivity under the tight comprehension condition, $R$ is also obtainable through $\gamma$-effectivity under $\chi$.

Proof. Let $(N, H, V)$ and $G=(N, S, A, g, U)$ be such that $a R b$ if and only if $u(a)$ dominates $u(b)$ in $(N, H, V)$ and $(N, V, H) \gamma$-corresponds to $G$ under the tight comprehension condition. Consider the game $\left(N, H, V_{\gamma}\right)$, where $V_{\gamma}(C)=\left\{x_{C} \in \chi\left(H_{C}\right): C\right.$ is $\gamma$-effective for $x_{C}$ in $\left.G\right\}$ for all coalitions $C$. Let $>$ and $>^{\prime}$ denote the dominance relations of $(N, H, V)$ and $\left(N, H, V_{\gamma}\right)$, respectively. Consider arbitrary $a, b \in A$.

First assume $a R b$. Then, $u(a)>_{C} u(b)$ for some coalition $C$. Then, there is some $x_{C} \in V(C)$ such that $x_{C} \geq u_{C}(a)>u_{C}(b)$. Observe that $V(C) \subseteq V_{\gamma}(C)$ and so $x_{C} \in V_{\gamma}(C)$. Hence, $u(a)>_{C}^{\prime}$ $u(b)$ and, subsequently, $u(a)>^{\prime} u(b)$.

For the opposite direction assume $u(a)>^{\prime} u(b)$. Then, there is some $x_{C} \in \chi(H)$ with $x_{C} \geq$ $u_{C}(a)>u_{C}(b)$ and $C$ is $\gamma$-effective for $x_{C}$. If $\gamma=\alpha$, there is some $s \in S$ such that for all $t \in S$, $u_{C}\left(g\left(s_{C}, t_{-C}\right)\right) \geq x_{C} \geq u_{C}(a)$. If $\gamma=\beta$, for all $s \in S$ there is some $t \in S$ such that $u_{C}\left(g\left(t_{C}, s_{-C}\right)\right) \geq$ $x_{C} \geq u_{C}(a)$. In either case, $C$ is $\gamma$-effective for $u_{C}(a)$. It follows that both $u(a)>_{C} u(b)$ and $u(a)>u(b)$. Hence, $a R b$, which concludes the proof. 


$$
\left[\begin{array}{ll}
(1,0,0) & (1,2,0) \\
(0,0,0) & (0,1,0)
\end{array}\right]\left[\begin{array}{ll}
(1,0,0) & (2,1,0) \\
(0,0,0) & (0,1,0)
\end{array}\right]
$$

Figure 2: A three-player game, in which player 1 chooses rows, player 2 chooses columns, and player 3 chooses matrices, showing that if the comprehension condition is tight, $\alpha$-effectivity does not generally imply superadditivity.

Characteristic functions based on either $\alpha$-effectivity or $\beta$-effectivity are perforce monotonic. However, if no restrictions are imposed on the comprehension conditions, superadditivity does not generally hold for characteristic functions obtained through either $\alpha$ - or $\beta$-effectivity. Even if comprehensiveness is assumed, only $\alpha$-effectivity guarantees superadditivity. ${ }^{3}$

Example 2. Consider the normal-form game depicted in Figure 2 and let $V$ be the characteristic function of the coalitional game $\alpha$-corresponding to it under the tight comprehension condition $\chi$. We find that both player 1 and player 2 are $\alpha$-effective for 1 , player 1 in virtue of the top row, player 2 because of the right column. Moreover, $1 \in V(\{1\})$ and $1 \in V(\{2\})$. Observe that the coalition $\{1,2\}$ consisting of player 1 and player 2 is $\alpha$-effective for neither $(1,2)$ nor $(2,1)$. Although, this coalition is $\alpha$-effective for the utility vector $(1,1)$-playing top row and right column would achieve this - there is no outcome that guarantees both players these utilities for which $\{1,2\}$ is $\alpha$-effective. Therefore, due to tightness of the comprehension condition, we merely have $V(\{1,2\})=\{(0,0),(1,0),(0,1)\}$ and superadditivity is not satisfied. It is worth observing that if full comprehensiveness were assumed, superadditivity would have been satisfied. This would have also been the case if $(1,2,0)$ and $(2,1,0)$ were both replaced by $(1,1,0)$.

Intuitively, superadditivity is a particularly natural property in the context of $\alpha$-effectivity. If two disjoint coalitions $C$ and $D$ can guarantee particular utilities to their members by playing particular strategies, then each member of either coalition should also be guaranteed that utility if both coalitions play those strategies simultaneously. Accordingly, examples like the above suggest that tight comprehension conditions are conceptually dubious in the context of $\alpha$-effectivity and that looser ones are more appropriate. As the topic does not affect the issues at hand, we will not pursue it here. Moreover, our model also allows for comprehension conditions which do guarantee superadditivity of NTU games obtained through $\alpha$-effectivity, , e.g., full comprehensiveness. We rather point at another important structural property related to superadditivity that all finite NTU games obtained through $\alpha$-effectivity satisfy. We will call a finite NTU game $(N, H, V) \alpha$-consistent if, for disjoint coalitions $C$ and $D, x_{C} \in V(C)$ and $y_{D} \in V(D)$ imply that there is some $u \in H$ such that $u_{C \cup D} \geq\left(x_{C}, y_{D}\right)$. Superadditivity is stronger than $\alpha$-consistency in that it additionally requires the coalition $C \cup D$ also to be effective for $u_{C \cup D}$. We find that every finite NTU game obtained through $\alpha$-effectivity satisfies the weaker property of $\alpha$-consistency.

Lemma 3. Let $\chi$ be a comprehension condition. Then, every finite NTU game that $\alpha$-corresponds to a normal-form game under $\chi$ is $\alpha$-consistent.

Proof. Let $(N, H, V)$ be an arbitrary finite NTU game and $G=(N, S, \Omega, g, U)$ be an equally arbitrary normal-form game such that $(N, H, V) \alpha$-corresponds to $G$. Let $C$ and $D$ be disjoint coalitions in $N$ with $x_{C} \in V(C)$ and $y_{D} \in V(D)$. Then, there are strategy profiles $s, t \in S$ such 
that for all $r \in S$ both $u\left(g\left(s_{C}, r_{-C}\right)\right) \geq x_{C}$ and $u\left(g\left(t_{D}, r_{-D}\right)\right) \geq y_{D}$. Let $\tilde{s}$ be a strategy profile defined such that $\tilde{s}_{C}=s_{C}$ and $\tilde{s}_{D}=t_{D}$. Then, for all $r \in S, u\left(g\left(\tilde{s}_{C \cup D}, r_{-(C \cup D)}\right)\right) \geq\left(x_{C}, y_{D}\right)$. Now observe that $u\left(g\left(\tilde{s}_{C \cup D}, r_{-(C \cup D)}\right)\right)$ is in $H$, and a fortiori also in $\chi(H)$, which concludes the proof.

\section{Dominance Relations through Coalitional Effectivity}

As it turns out, the class of finite NTU games obtainable through $\alpha$-effectivity and the class of games obtainable through $\beta$-effectivity are not identical. Theorem 2 shows that a restriction to the latter class of games imposes no constraints on the dominance relations that are obtainable in addition to their being irreflexive. On the other hand, we find $\alpha$-effectivity only yields dominance relations that also satisfy the edge-mapping property, which is defined in Section 5.2.

\subsection{Dominance Relations through $\beta$-Effectivity}

We start by proving that every irreflexive relation on a set of outcomes $A$ is obtainable through $\beta$-effectivity and vice versa.

Theorem 2. Let $R$ be a binary relation on a finite set $A$ and $\chi$ an arbitrary comprehension condition. Then, $R$ is obtainable through $\beta$-effectivity under $\chi$ if and only if $R$ is irreflexive.

Proof. If $|A|=1$ the proof is trivial and for the remainder of the proof we will assume that $A$ contains at least two outcomes. The only-if direction is also trivial, as the dominance relation of any finite NTU game is irreflexive.

For the opposite direction, by virtue of Lemma 2, it suffices to give the proof for the case in which $\chi$ is tight. Consider an arbitrary irreflexive relation $R$ on a set $A$ along with a particular finite NTU game $V_{R}=(N, H, V)$ in the collection of games for $R$ as defined in the proof of Theorem 1. The set $N$ of players and the set $H$ of feasible outcomes are fixed by $R$, but we still have to define $V$. Let $A$ be enumerated as $a_{1}, \ldots, a_{|A|}$. Then, for each $u \in H$ there is some $1 \leq m \leq|A|$ such that $u=u\left(a_{m}\right)$. For each player $i \in N$ we define an outcome $a_{i}^{*} \in A$ as follows.

For each $a \in A$, let,

$$
a_{i_{a}}^{*}=a_{j_{a}}^{*}=a_{k}, \quad \text { where } \quad k= \begin{cases}\min \left\{m: a R a_{m}\right\} & \text { if } a R b \text { for some } b \in A, \\ \min \left\{m: a_{m} \neq a\right\} & \text { otherwise. }\end{cases}
$$

Observe that $u_{i}\left(a_{i}^{*}\right)=\min _{u \in H} u_{i}$ for each $i \in N$. Define for each coalition $C$,

$$
A_{C}^{*}=\bigcup_{i \in C}\left\{a \in A: u_{C}(a) \leq u_{C}\left(a_{i}^{*}\right)\right\}
$$

and set

$$
X_{C}^{*}=\left\{u_{C}(a): a \in A_{C}^{*}\right\} .
$$

Obviously, $X_{C}^{*} \subseteq H_{C}^{*}$ for each coalition $C$. Then, set for each coalition $C$ in $N$,

$$
V(C)= \begin{cases}H_{C} & \text { if }\left\{i_{a}, j_{a}\right\} \subseteq C, \text { for some } a \in A \\ X_{C}^{*} & \text { otherwise }\end{cases}
$$


Defined thus, $V_{R}$ is clearly in the collection of games for $R$ as defined in the proof of Theorem 1.

It suffices to prove that $V_{R}$ can be obtained through $\beta$-effectivity. To this end we construct a normal-form game $G_{\beta}^{R}$, which through $\beta$-effectivity induces a unique finite NTU game $V_{\beta}^{R}$. We then show that $V_{R}=V_{\beta}^{R}$. Define $G_{\beta}^{R}=(N, S, \Omega, g, U)$ with $\Omega=A, N=\left\{i_{a}, j_{a}: a \in A\right\}$, and $U=\left(u_{i j}\right)_{i \in N, j \in A}$ as in the proof of Theorem 1. Moreover, for each player $i$ in $N$, we have $S_{i}=\{0,1\} \times\{0,1\} \times\{1, \ldots,|A|\}$, with representative element $s_{i}=\left(s_{i}^{1}, s_{i}^{2}, s_{i}^{3}\right)$. This leaves us with the definition of the outcome function $g$. Suppose strategy profile $s$ is played. Intuitively, the coalition $C(s)$ consisting of all pairs $i$ and $j$ with $s_{i}^{1}=s_{j}^{1}=1$ is then formed. Formally, we define

$$
C(s)=\bigcup_{a \in A}\left\{i \in\left\{i_{a}, j_{a}\right\}: s_{i_{a}}^{1}=s_{j_{a}}^{1}=1\right\} .
$$

The members of $C(s)$ then decide whether the game is continued by all players in $N$ playing the modulo game $M(A,|A|)$ or the modulo game $M\left(A_{-C(s)}^{*},|A|\right)$. The latter is played if $s_{i}^{2}=0$ for all $i \in C(s)$ and $C(s) \neq N$, the former, otherwise. Observe that this also covers the case in which $C(s)=\emptyset$. Depending on which modulo game is played, the outcome is then determined by $s^{3}=\left(s_{i}^{3}\right)_{i \in N}$. Let for each non-empty $B \subseteq A$ the function $\phi_{B}: \mathbb{N}^{N} \rightarrow N$ be defined such that for $x=\left(x_{i}\right)_{i \in N} \in \mathbb{N}^{N}$,

$$
\phi_{B}(x)=1+\left(\sum_{i \in N} x_{i} \bmod |B|\right) .
$$

Formally, we define the outcome function $g$ such that for all strategy profiles $s \in S$,

$$
g(s)=a_{m}, \quad \text { where } \quad m= \begin{cases}\phi_{A}\left(s^{3}\right) & \text { if } C(s)=N \text { or } s_{i}^{2}=1 \text { for some } i \in C(s), \\ \phi_{B}\left(s^{3}\right) & \text { for } B=A_{-C(s)}^{*}, \text { otherwise. }\end{cases}
$$

Let $H^{\prime}=\{u(g(s)): s \in S\}$ and let

$$
V_{\beta}(C)=\left\{x_{C} \in H_{C}: C \text { is } \beta \text {-effective for } x_{C} \text { in } G_{\beta}^{R}\right\} .
$$

Define $V_{\beta}^{R}=\left(N, H^{\prime}, V_{\beta}\right)$. Then, having assume the comprehension condition $\chi$ to be tight, $V_{\beta}^{R}$ clearly $\beta$-corresponds to $G_{\beta}^{R}$ under $\chi$.

To show that $V_{\beta}^{R}=V_{R}$, we first prove that $H=H^{\prime}=\{u(g(s)): s \in S\}$. As it is obvious that $\{u(g(s)): s \in S\} \subseteq H$, consider an arbitrary $u \in H$. Then, $u=u\left(a_{m}\right)$ for some $1 \leq m \leq|A|$. Now consider the strategy profile $\left(s_{i}\right)_{i \in N}$ such that

$$
s_{i}= \begin{cases}(1,1, m) & \text { if } i=1 \\ (1,1,|A|) & \text { otherwise }\end{cases}
$$

Now $C(s)=N$ and, informally, the modulo game $M(A,|A|)$ is played. Hence, $g(s)=\phi_{A}\left(s^{3}\right)=a_{m}$ and $u\left(a_{m}\right) \in\{u(g(s)): s \in S\}$, as desired.

It remains to be shown that for each coalition $C$ in $N, V(C)=V_{\beta}(C)$. To this end, first consider an arbitrary coalition $C$ containing a pair $i^{*}$ and $j^{*}$. Then, $V(C)=H_{C}$. Having assumed the comprehension condition $\chi$ to be tight, trivially, $V_{\beta}(C) \subseteq H_{C}$. To show that also $H_{C} \subseteq V_{\beta}(C)$, 
consider an arbitrary $u \in H$. There is some $1 \leq m \leq|A|$ such that $u=u\left(a_{m}\right)$. Now consider an arbitrary strategy profile $s=\left(s_{i}\right)_{i \in N}$. Let $t=\left(t_{i}\right)_{i \in N}$ be the strategy profile such that for all $i \in N$

$$
t_{i}= \begin{cases}\left(1,1, m^{\prime}\right) & \text { if } i=i^{*}, \\ \left(1,1, s_{i}^{3}\right) & \text { otherwise. }\end{cases}
$$

where $m^{\prime}$ satisfies

$$
m=1+\left(\left(m^{\prime}+\sum_{i \neq i^{*}} s_{i}^{3}\right) \bmod |A|\right) .
$$

Because $t_{i^{*}}^{1}=t_{j^{*}}^{1}=1$, we have $\left\{i^{*}, j^{*}\right\} \subseteq C\left(t_{C}, s_{-C}\right)$. As $t_{i^{*}}^{2}=1$, moreover, $\left(t_{C}, s_{-C}\right)$ leads to the modulo game $M(A,|A|)$ being played. By choice of $m^{\prime}$, this leads to outcome $a_{m}$ to come about. Formally, it is also easily appreciated that $g\left(t_{C}, s_{-C}\right)=a_{m}$. Hence, $C$ is $\beta$-effective for $u\left(a_{m}\right)=x_{C}$ and $x_{C} \in V_{\beta}(C)$, as desired.

Finally, consider an arbitrary coalition $C$ that contains no pairs, i.e., for no $a \in A,\left\{i_{a}, j_{a}\right\} \subseteq C$. Also consider an arbitrary $x_{C} \in \mathbb{R}^{C}$. First assume that $x_{C} \in V(C)$. Then, $x_{C}=u_{C}\left(a_{m}\right)$ for some $1 \leq m \leq|A|$ and $x_{C} \leq u_{C}\left(a_{i^{*}}^{*}\right)$ for some $i^{*} \in C$. Consider an arbitrary strategy profile $s \in S$ and define a strategy profile $t=\left(t_{i}\right)_{i \in N}$ such that $t_{j}=s_{j}$ for all $j \neq i^{*}$ and $t_{i^{*}}=\left(0,0, m^{\prime}\right)$, where $m^{\prime}$ satisfies

$$
m=1+\left(\left(m^{\prime}+\sum_{i \neq i^{*}} s_{i}^{3}\right) \bmod |B|\right),
$$

for $B=A$ if $s_{i}^{2}=1$ for some $i \in C(s)$ and $B=A_{-C(s)}^{*}$, otherwise. Because $t_{i^{*}}^{1}=0$ we have $i^{*} \notin C\left(s_{-C}, t_{C}\right)$. Hence, $\left(s_{-C}, t_{C}\right)$ leads to some modulo game $M(B,|A|)$ being played with $a_{m} \in B$. Moreover, the choice of $m^{\prime}$ guarantees $a_{m}$ as the outcome. Formally, it is easily checked that $g\left(s_{-C}, t_{C}\right)=a_{m}$. It follows that $x_{C}=u_{C}\left(a_{m}\right) \in V_{\beta}(C)$.

For the opposite direction, assume $x_{C} \in V_{\beta}(C)$. Let $D$ be the set of partners of the members in $C$, i.e.,

$$
D=\{j \in N: j \text { is the partner of some } i \in C\} .
$$

Because $C$ contains no pairs, $C$ and $D$ are disjoint. Moreover, $\left\{a_{i}^{*}: i \in C\right\}=\left\{a_{i}^{*}: i \in D\right\}$. Also $u_{C \cup D}(a) \geq u_{C \cup D}(b)$ implies $u_{C}(a) \geq u_{C}(b)$ for all $a, b \in A$. Hence, $A_{C \cup D}^{*} \subseteq A_{C}^{*}$. Let further $E=N \backslash(C \cup D)$. Thus, $E$ only contains pairs, i.e., if $i$ and $j$ are partners, then $i \in E$ if and only if $j \in E$. Now let $s=\left(s_{i}\right)_{i \in N}$ be the strategy profile such that

$$
s_{i}= \begin{cases}(1,1,1) & \text { if } i \in E, \\ (0,1,1) & \text { otherwise. }\end{cases}
$$

Then, informally, if the players in $E \cup D$ adhere to $s$, no matter which joint strategy $C$ adopts, the coalition $E$ will be formed and the modulo game $M\left(A_{C \cup D}^{*},|A|\right)$ will played. Because $x_{C} \in V_{\beta}(C)$, there is some strategy profile $t=\left(t_{i}\right)_{i \in N}$ such that $u_{C}\left(g\left(s_{-C}, t_{C}\right)\right) \geq x_{C}$. Observe that $g\left(s_{-C}, t_{C}\right) \in$ $A_{C \cup D}^{*}$ and, since $A_{C \cup D}^{*} \subseteq A_{C}^{*}$, also $g\left(s_{-C}, t_{C}\right) \in A_{C}^{*}$. By tightness of $\chi$ and $C$ containing no pairs, there is some $b \in A$ with $x_{C}=u_{C}(b)$ and, therefore, $b \in A_{C}^{*}$ as well. Hence, $u_{C}(b) \in X_{C}^{*}$ and we may conclude that $x_{C} \in V(C)$. 


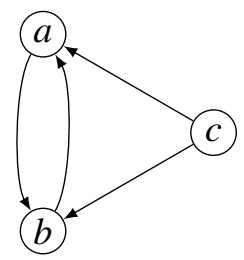

$\left[\begin{array}{lll}(1,1) & (1,1) & (1,1) \\ (1,1) & (1,0) & (0,1) \\ (1,1) & (0,1) & (1,0)\end{array}\right]$

Figure 3: Example showing that it can occur that two outcomes dominate each another in a graph obtained through $\alpha$-effectivity, where $u(a)=(1,0), u(b)=(0,1)$ and $u(c)=(1,1)$. In the two-player non-cooperative game depicted on the right player 1 chooses rows and player 2 columns.

\subsection{Edge-Mappings and the Edge-Mapping Property}

For dominance graphs obtained through $\alpha$-effectivity matters are slightly more complicated than for those obtained through $\beta$-effectivity. For instance, it is not the case that every irreflexive dominance relation can be obtained through $\alpha$-effectivity. To appreciate this, consider the dominance graph on two alternatives $a$ and $b$ such that $a$ and $b$ dominate each other, i.e., $a>b$ as well as $b>a$. Now assume for a contradiction that this graph can be induced through $\alpha$ effectivity. As $a>b$, there must be some coalition $C$ that is $\alpha$-effective for outcome $a$ and such that all of its members strictly prefer outcome $a$ to outcome $b$, i.e., $u_{C}(a)>u_{C}(b)$. Similarly, because $b>a$ there is some coalition $D$ that is $\alpha$-effective for outcome $b$ and $u_{D}(b)>u_{D}(a)$. It follows that $C$ and $D$ are disjoint. Moreover, by $\alpha$-consistency, there is some outcome $c$ such that $u_{C \cup D}(c) \geq\left(u_{C}(a), u_{D}(b)\right)$. Clearly, $c$ has to be distinct from both $a$ and $b$, a contradiction.

On the other hand, a dominance graph containing alternatives that dominate each other does not preclude that dominance relation being obtainable through $\alpha$-effectivity. Consider, for instance, the dominance graph on three alternatives, $a, b$ and $c$, depicted in Figure 3. There alternative $a$ dominates alternative $b$ and alternative $b$ dominates alternative $a$. Nevertheless, the graph is obtainable through $\alpha$-effectivity from the non-cooperative game depicted on the right.

If a dominance relation $>$ is obtained through $\alpha$-effectivity from a normal-form game, it is worth remarking that $a>_{C} b$ does not so much mean that coalition $C$ has a strategy that, no matter which strategies the other players adopt, $a$ is the outcome of the game. Rather, $a>_{C} b$ signifies that in the normal-form game coalition $C$ has a strategy at her disposal which guarantees, irrespective of the strategies the other players adopt, that the outcome falls within a set of outcomes each of which is at least as good for the members of $C$ as $a$ and strictly better than $b$. Accordingly, it is possible to associate with each edge $(a, b)$ in the dominance relation $>$ a socalled witnessing coalition $C$, along with such a set of outcomes that are at least as good as $a$ and strictly better than $b$ for all members in $C$. Moreover, for all distinct edges $(a, b)$ and $(c, d)$ with disjoint witnessing coalitions these sets of outcomes should have a non-empty intersection. Otherwise the witnessing coalitions of $(a, b)$ and $(c, d)$ could each play a strategy that guarantees the outcome to fall within disjoint sets, which is impossible. Accordingly, if a binary relation $R$ is obtainable through $\alpha$-effectivity, it must at least be possible to associate with each edge $(a, b)$ a set of outcomes containing $a$ but not $b$. Moreover, any such so-called edge mapping has to satisfy a number of consistency conditions. For instance, if both $(a, b)$ and $(b, a)$ are edges in $R$, they 
have disjoint witnessing coalitions. Hence, the edge mapping should associate overlapping sets of outcomes with $(a, b)$ and $(b, a)$. The full set of consistency conditions is summarized in the edge-mapping property below. We will see that binary relations obtainable through $\alpha$-effectivity are completely characterized by irreflexivity and the edge-mapping property.

Formally, we define an edge mapping for a given irreflexive binary relation $R$ on a set $A$ as a function $\psi: A \times A \rightarrow 2^{A}$ such that for each edge $(a, b) \in R$ we have $a \in \psi(a, b)$ and $b \notin \psi(a, b)$. Observe that an edge mapping $\psi$ is not commutative. Rather, in general $\psi(a, b) \neq \psi(b, a)$. Given an edge mapping $\psi$ for $R$, we say that two edges $(a, b)$ and $(c, d)$ in $R$ are $\psi$-exclusive whenever at least one of the following two conditions holds:

(i) $\{a, b\} \cap \psi(c, d) \neq \emptyset$ and $d \in \psi(a, b)$,

(ii) $\{c, d\} \cap \psi(a, b) \neq \emptyset$ and $b \in \psi(c, d)$.

In this context it is worth observing that for every asymmetric relation there is an edge mapping $\psi$ such that no two edges are $\psi$-exclusive. Merely set $\psi(a, b)=\{a\}$. Then, for all edges $(a, b)$ and $(c, d)$ such that $\{a, b\} \cap \psi(c, d) \neq \emptyset$, either $a=c$ or $b=c$. If the former we have $d \neq a$ immediately, if the latter this follows by asymmetry. In either case $d \notin \psi(a, b)$ (also see Corollary 1, below). On the other hand, for any alternatives $a, b$ in $A$, if both $(a, b) \in R$ and $(b, a) \in R,(a, b)$ and $(b, a)$ are $\psi$-exclusive for any edge mapping $\psi$. Intuitively, $(a, b)$ and $(c, d)$ being $\psi$-exclusive means that the witnessing coalitions of $(a, b)$ and $(c, d)$ have to be disjoint given $\psi$. To appreciate this, recall the intuitive interpretation of $\psi(a, b)$ as a set of outcomes that are at least as desirable as $a$ and strictly more desirable than $b$ for the members of the coalition witnessing $(a, b)$. Now assume $(i)$ to hold but that some player $i$ is member of both the witnessing coalition for $(a, b)$ as well as the one witnessing $(c, d)$. As $\{a, b\} \cap \psi(c, d) \neq \emptyset$, then

$$
u_{i}(a)>u_{i}(b) \geq u_{i}(c)>u_{i}(d) .
$$

Hence, $d \notin \psi(a, b)$ and a contradiction follows, meaning that the witnessing coalitions of $(a, b)$ and $(c, d)$ have to be disjoint. An analogous argument holds for (ii). This idea is made precise in Lemma 5, below.

The edge-mapping property is then defined as follows.

Definition 5 (Edge-mapping property). Let $R \subseteq A \times A$ a binary relation on a set $A$. $R$ is said to satisfy the edge-mapping property $(E M P)$ if an edge mapping $\psi: A \times A \rightarrow 2^{A}$ exists such that $\bigcap_{(a, b) \in R^{\prime}} \psi(a, b) \neq \emptyset$ for each subrelation $R^{\prime} \subseteq R$ of which the edges are pairwise $\psi$-exclusive.

Informally, the edge-mapping property guarantees that disjoint witnessing coalitions under the edge-mapping $\psi$ cannot force the game to end in different outcomes.

Example 3. Consider the three binary relations, $R_{1}, R_{2}$ and $R_{3}$ depicted in Figure 4 . Only $R_{1}$ satisfies the edge-mapping property in virtue of the edge mapping $\psi$ summarized in the table below.

\begin{tabular}{ll}
$(x, y)$ & $\psi(x, y)$ \\
\hline$(a, b)$ & $\{a, d\}$ \\
$(a, c)$ & $\{a, d\}$ \\
$(b, a)$ & $\{b, d\}$ \\
$(d, b)$ & $\{d\}$ \\
$(d, c)$ & $\{d\}$
\end{tabular}




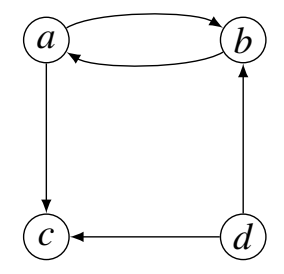

$\left(R_{1}\right)$

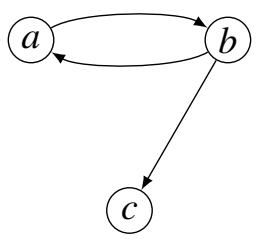

$\left(R_{2}\right)$
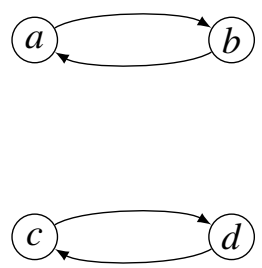

$\left(R_{3}\right)$

Figure 4: Of the three binary relations depicted, only $R_{1}$ satisfies the edge-mapping property.

Then $d \in \psi(x, y)$ for all $x, y \in\{a, b, c, d\}$ and, therefore, cannot fail to satisfy EMP. In $R_{2}$, the edges $(a, b)$ and $(b, a)$ are clearly $\psi$-exclusive for every edge mapping $\psi$, as $b \in \psi(b, a)$ and $a \in \psi(a, b)$. Suppose $\psi$ were an edge mapping with respect to which $R_{2}$ satisfies EMP. In that case, $\psi(a, b)=\{a, c\}, \psi(b, a)=\{b, c\}$, and $b \in \psi(b, c)$. Now $(a, b)$ and $(b, c)$ are $\psi$-exclusive because $c \in \psi(a, b)$ and $b \in \psi(b, c)$ whereas $(b, a)$ and $(b, c)$ are $\psi$-exclusive because $b \in \psi(b, c)$ and $c \in \psi(b, a)$. As, however, $a \notin \psi(b, a), b \notin \psi(a, b)$ and $c \notin \psi(b, c), \psi(a, b) \cap \psi(b, a) \cap(b, c)=\emptyset$. Hence, $R_{2}$ does not satisfy EMP. We leave it to the avid reader to verify that $R_{3}$ does not satisfy EMP either.

We find that the dominance relation of every $\alpha$-consistent finite NTU game satisfies the edge-mapping property.

Lemma 4. The dominance relation of every $\alpha$-consistent finite NTU game satisfies the edgemapping property.

Proof. Let $>$ be the dominance relation of an $\alpha$-consistent finite NTU game $(N, H, V)$. For all $u, v \in H$ with $u>v$, there is some coalition $C(u, v)$ such that $u_{C(u, v)}>v_{C(u, v)}$. Moreover, there is some $x \in V(C(u, v))$ with $x_{C(u, v)} \geq u_{C(u, v)}>v_{C(u, v)}$. Now define $\psi: H \times H \rightarrow 2^{H}$ such that, for all $u, v \in H$,

$$
\psi(u, v)=\left\{x \in H: x_{C(u, v)} \geq u_{C(u, v)}>v_{C(u, v)}\right\} .
$$

Obviously, $\psi$ is an edge mapping for the dominance relation $>$, as for all $u, v \in H$ we have $u \in$ $\psi(u, v)$ and $v \notin \psi(u, v)$. Let $u^{1}, v^{1}, \ldots, u^{m}, v^{m} \in H$ such that $\left(u^{1}, v^{1}\right), \ldots,\left(u^{m}, v^{m}\right)$ are pairwise $\psi$ exclusive edges in the dominance relation $>$. For each $1 \leq k \leq m$, we have $C_{k}$ denote the coalition $C\left(u^{k}, v^{k}\right)$.

First, we establish that the coalitions $C_{i}$ and $C_{j}$ are disjoint, for all $1 \leq i<j \leq m$. Without loss of generality we may assume that $\left\{u^{i}, v^{i}\right\} \cap \psi\left(u^{j}, v^{j}\right) \neq \emptyset$ and $v^{j} \in \psi\left(u^{i}, v^{i}\right)$. Now observe that the former implies that $u_{C_{j}}^{i}>v_{C_{j}}^{j}$ or $v_{C_{j}}^{i}>v_{C_{j}}^{j}$, whereas the latter yields both $v_{C_{i}}^{j} \geq u_{C_{i}}^{i}$ and $v_{C_{i}}^{j} \geq v_{C_{i}}^{i}$. Hence, $C_{i} \cap C_{j}=\emptyset$. We now show that $\bigcap_{1 \leq i \leq m} \psi\left(u^{i}, v^{i}\right) \neq \emptyset$. For each $1 \leq k \leq m$ there is some $x_{C_{k}} \in V\left(C_{k}\right)$ such that $x_{C_{k}} \geq u_{C_{k}}^{k}>v_{C_{k}}^{k}$. Because $C_{1}, \ldots, C_{k}$ are pairwise disjoint and $V$ is $\alpha$-consistent, there is some $u^{*} \in H$ such that $u_{C_{1} \cup \ldots \cup C_{m}}^{*} \geq x_{C_{1} \cup \ldots \cup C_{m}}$. It follows that $u^{*} \in \psi\left(u^{i}, v^{i}\right)$ for all $1 \leq i \leq m$. Hence, $\bigcap_{1 \leq i \leq m} \psi\left(u^{i}, v^{i}\right) \neq \emptyset$, which concludes the proof. 
With Lemma 3, every finite NTU game obtained through $\alpha$-effectivity is $\alpha$-consistent. Hence, Lemma 4 implies that the dominance relation of any such game satisfies the edge-mapping property.

\subsection{Dominance Relations through $\alpha$-Effectivity}

In order to obtain a full characterization of the dominance relation that can be obtained through $\alpha$-effectivity, we construct for each irreflexive relation $R$ with the edge-mapping property a noncooperative game $G_{\alpha}^{R}$. We then show that $R$ is induced as the dominance relation of the NTU game that $\alpha$-corresponds to $G_{\alpha}^{R}$. The players of this game are defined by the weak (i.e., reflexive, transitive and complete) orders over the outcome set $A$. Thus, by contrast to the constructions used in the proofs of Theorems 1 and 2, the number of players is exponential, rather than linear, in the number of vertices.

Let $\mathscr{W}(A)$ denote the set of weak orders $P$ over the set $A$. We write $a \gtrsim_{P} b$ to signify that $(a, b) \in P$. Also $a \sim_{P} b$ denotes that both $a \gtrsim_{P} b$ and $b \gtrsim_{P} a$, and $a>_{P} b$ that $a \gtrsim_{P} b$ but not $b \gtrsim_{P} a$. Then, define, for each $X \subseteq A$ with $a \in X$ and $b \notin X$,

$$
C(X, a, b)=\left\{P \in \mathscr{W}(A): x \gtrsim_{P} a>_{P} b \text { for all } x \in X\right\} .
$$

If an edge mapping $\psi$ is fixed in the context we will denote $C(\psi(a, b), a, b)$ by $C_{\psi}(a, b)$, even omitting the subscript if $\psi$ is clear from the context. Before we give our characterization result, we first prove a lemma, establishing the exact conditions under which two coalitions $C_{\psi}(a, b)$ and $C_{\psi}(c, d)$ are disjoint.

Lemma 5. Let $\psi$ be an edge mapping for an irreflexive relation $R$ on $A$. Then, for all $(a, b),(c, d) \in$ $R$,

$$
C_{\psi}(a, b) \cap C_{\psi}(c, d)=\emptyset \text { if and only if }(a, b) \text { and }(c, d) \text { are } \psi \text {-exclusive. }
$$

Proof. For the if-direction assume $(a, b)$ and $(c, d)$ to be $\psi$-exclusive. Without loss of generality we may assume that $\{a, b\} \cap \psi(c, d) \neq \emptyset$ and $d \in \psi(a, b)$. From the former follows that either $a>_{P} d$ or $b>_{P} d$ for each $P \in C(c, d)$, whereas the latter yields both $d \gtrsim_{P} a$ and $d \gtrsim_{P} b$ for each $P \in C(a, b)$. Hence, $C(a, b) \cap C(c, d)=\emptyset$.

For the opposite direction assume that $(a, b)$ and $(c, d)$ are not $\psi$-exclusive. Then,

(i) $d \in \psi(a, b)$ implies $\{a, b\} \cap \psi(c, d)=\emptyset$, and

(ii) $b \in \psi(c, d)$ implies $\{c, d\} \cap \psi(a, b)=\emptyset$.

We distinguish three cases: (1) $d \in \psi(a, b)$, (2) $b \in \psi(c, d)$ and (3) neither $d \in \psi(a, b)$ nor $b \in \psi(c, d)$. First assume $d \in \psi(a, b)$. Then, $d \neq b$. By $(i)$, moreover, $\{a, b\} \cap \psi(c, d)=\emptyset$. Hence, $a \neq c$ and $b \notin \psi(c, d)$. Therefore, there exists a weak order $P$ on $A$ such that for all $x \in(\psi(a, b) \cup \psi(c, d)) \backslash\{a, d\}$,

$$
x>_{P} a \sim_{P} d>_{P} b .
$$

Observe that both $P \in C(a, b)$ and $P \in C(c, d)$. Hence, $C(a, b) \cap C(\psi, c, d) \neq \emptyset$. Case (2) is covered by an analogous argument. 
Finally, in case (3), we have $a \neq b, c \neq d, a \neq d$ as well as $b \neq c$. Moreover, $\{b, d\} \cap$ $(\psi(a, b) \cup \psi(c, d))=\emptyset$. It follows that there is a weak order $P$ on $A$ such that for all $x \in$ $(\psi(a, b) \cup \psi(c, d)) \backslash\{a, c\}$,

$$
x>_{P} a \sim_{P} c>_{P} b \sim_{P} d .
$$

Then, both $P \in C(a, b)$ and $P \in C(c, d)$ and we may conclude $C(a, b) \cap C(c, d) \neq \emptyset$.

We are now in a position to prove our characterization result for dominance graphs obtained through $\alpha$-effectivity. Even if the edge-mapping property may appear a bit contrived, it is a property of binary relations that is defined independently of their interpretation as dominance relations. Moreover, Theorem 3 can be used to obtain more intuitive results. Three of these are captured in Corollaries 1, 2, and 3.

Theorem 3. Let $R$ be a binary relation on a finite set $A$ and $\chi$ a comprehension condition. Then, $R$ is obtainable through $\alpha$-effectivity under $\chi$ if and only if $R$ is irreflexive and satisfies the edge-mapping property.

Proof. The only-if direction is an immediate consequence of Lemma 3 and Lemma 4: Every finite NTU game that $\alpha$-corresponds to some normal-form game under $\chi$ is $\alpha$-consistent, and the dominance relation of every $\alpha$-consistent finite NTU game satisfies the edge-mapping property.

For the opposite direction, assume $R$ to satisfy the edge-mapping property and let $\psi: A \times A \rightarrow$ $2^{A}$ be the witnessing edge mapping. We first construct a normal-form game $G_{\alpha}^{R}=(N, S, \Omega, g, U)$, where the set of players $N$ is given by the set $\mathscr{W}(A)$ of weak orders over $A$ and $\Omega=A=$ $\left\{a_{1}, \ldots, a_{|A|}\right\}$. By virtue of Lemma 2 we may assume that $\chi$ is tight. Each player $P$ defines his own preference relation over $A$, i.e., $u_{P}(a) \geq u_{P}(b)$ if and only if $a \gtrsim_{P} b$. We have the utility matrix $U=\left(u_{i j}\right)_{i \in N, j \in A}$ represent these preferences. By $\mathscr{C}(R)$ we denote the set $\left\{C_{\psi}(a, b): a R b\right\}$. In the remainder of the proof we will omit the subscript $\psi$ in $C_{\psi}(a, b)$. For each player $i \in N$, the set $S_{i}$ of strategies is defined as $S_{i}=A \times A \times\{1, \ldots,|A|\}$, with typical element $s_{i}=\left(s_{i}^{1}, s_{i}^{2}, s_{i}^{3}\right)$.

This leaves us with the definition of the outcome function $g: S \rightarrow A$. For a better understanding, however, we first give an informal description of the game $G_{\alpha}^{R}$ and introduce a number of notational conventions. The game $G_{\alpha}^{R}$ can be understood as follows. By choosing the strategies $s_{i}^{1}$ and $s_{i}^{2}$ a player announces which coalition $C(x, y)$, where $x, y \in A$ and $x R y$, he wishes to belong to. Only if all members of a coalition express the wish to belong to that very coalition, it is actually formed. In this way the simultaneous formation of overlapping coalitions is precluded. Then, a modulo game $M(X,|A|)$ is played, where $X \subseteq A$ and the outcome of which is determined by $\left(s_{i}^{3}\right)_{i \in N}$. The resulting outcome is also the outcome of $G_{\alpha}^{R}$. Accordingly, each coalition $C(a, b)$ in $\mathscr{C}(R)$ can force the outcome of the game to fall within the set $\psi(a, b)$ by choosing an appropriate joint strategy. This guarantees the members $i$ of $C(a, b)$ a utility of minimally $u_{i}(a)$, no matter which strategies the other players adopt.

Formally, for each strategy profile $s=\left(s^{1}, s^{2}, s^{3}\right)$ in $S$, we say that $C(a, b)$ forms at $s$ if $s_{i}^{1}=a$ and $s_{i}^{2}=b$ for all $i \in C(a, b)$. Now define $\mathscr{C}(s)$ as the set of coalitions in $\mathscr{C}(R)$ that form at $s$, i.e.,

$$
\mathscr{C}(s)=\{C(a, b) \in \mathscr{C}(R): C(a, b) \text { forms at } s\} .
$$

Defined thus, all coalitions in $\mathscr{C}(s)$ are pairwise disjoint. Moreover, by virtue of Lemma 5, for all $a, b, c, d \in A$ with $C(a, b)$ and $C(c, d)$ distinct coalitions in $\mathscr{C}(s)$, the edges $(a, b),(c, d) \in R$ are 
$\psi$-exclusive. With each strategy profile $s=\left(s^{1}, s^{2}, s^{3}\right)$ we associate a set $X(s) \subseteq A$ of outcomes defined as,

$$
X(s)=\bigcap\{\psi(a, b): C(a, b) \in \mathscr{C}(s)\} .
$$

We postulate that $X(s)=A$ in case $\mathscr{C}(s)=\emptyset$. As $R$ satisfies the edge-mapping property, for each strategy profile $s$ the set $X(s)$ is non-empty.

We are now in a position to formally define the outcome function $g$, such that for all strategy profiles $s=\left(s^{1}, s^{2}, s^{3}\right)$ in $S$,

$$
g(s)=a_{m}, \quad \text { where } \quad m=1+\left(\sum_{i \in N} s_{i}^{3} \bmod |X(s)|\right) .
$$

Accordingly, by merely forming, each coalition $C(a, b)$ in $\mathscr{C}(R)$ has a strategy that guarantees the outcome of $G_{\alpha}^{R}$ to fall within $\psi(a, b)$, no matter which strategies the other players adopt.

Let $V_{\alpha}^{R}$ be the finite NTU game $\left(N, H, V_{\alpha}\right)$ where $H=\{u(g(s)): s \in S\}$ and for each coalition $C$ in $N$,

$$
V_{\alpha}(C)=\left\{x_{C} \in H_{C}: C \text { is } \alpha \text {-effective for } x_{C} \text { in } G_{\alpha}^{R}\right\} .
$$

Having assumed the comprehension condition $\chi$ to be tight, $V_{\alpha}^{R}$ clearly $\alpha$-corresponds to $G_{\alpha}^{R}$, so it remains to be shown that $V_{\alpha}^{R}$ induces $R$.

Observe that $u(a)=u(b)$ if and only if $a=b$. The if-direction is trivial. For the other direction, observe that there is some weak order $P \in \mathscr{W}(A)$ such that $a>_{P} b$. As $P \in N$, also $u_{P}(a)>u_{P}(b)$ signifying that $a \neq b$. Consequently, $|H|=|A|$ and it suffices to prove that for all $a, b \in A, a R b$ if and only if $u(a)>u(b)$.

Consider arbitrary $a, b \in A$ and assume $a R b$. Then, $C(a, b) \in \mathscr{C}(R)$. Let $s=\left(s_{i}\right)_{i \in N}$ be strategy profile such that $s_{i}=(a, b, 1)$ for all $i \in C(a, b)$ and consider an arbitrary strategy profile $t=\left(t_{i}\right)_{i \in N}$. Let $s^{*}=\left(s_{C(a, b)}, t_{-C(a, b)}\right)$. Then, $C(a, b)$ is formed at $s^{*}$, i.e., $C(a, b) \in$ $\mathscr{C}\left(s^{*}\right)$. Hence, $X\left(s^{*}\right) \subseteq \psi(a, b)$ and thus $g\left(s^{*}\right) \in \psi(a, b)$. Moreover, $u_{C(a, b)}\left(g\left(s^{*}\right)\right) \geq u_{C(a, b)}(a)>$ $u_{C(a, b)}(b)$. As $t$ had been chosen arbitrarily, it follows that $u_{C(a, b)}(a) \in V_{\alpha}(C(a, b))$. Therefore, $u(a)$ dominates $u(b)$ via $C(a, b)$, which yields $u(a)>u(b)$.

For the opposite direction, assume that $a>b$. Clearly, $a \neq b$. Also, there is some coalition $C$ in $N$ and some strategy profile $\tilde{s} \in S$ such that,

$$
u_{C}\left(g\left(\tilde{s}_{C}, t_{-C}\right)\right) \geq u_{C}(a)>u_{C}(b), \quad \text { for all } t \in S .
$$

observe that $C \neq N$-otherwise $C$ would also have contained weak orders $P$ with $u_{P}(b) \geq$ $u_{P}(a)$-and let $i^{*} \in N \backslash C$. Now assume for a contradiction that it is not the case that $a R b$ and define for each $1 \leq k \leq|A|$ a strategy profile $s[k]=\left(s[k]_{i}\right)_{i \in N}$ such that for all $i \in N$

$$
s[k]_{i}= \begin{cases}(a, b, k) & \text { if } i=i^{*}, \\ \left(a, b, \tilde{s}_{i}^{3}\right) & \text { otherwise. }\end{cases}
$$

Observe that for each strategy profile $s=\left(s^{1}, s^{2}, s^{3}\right)$, the set $\mathscr{C}(s)$ of coalitions that form under $s$ as well as the set $X(s)$ only depend on the first two components $s^{1}$ and $s^{2}$ of $s$. Accordingly, there is some set of disjoint coalitions $\mathscr{C}^{*}$ and some set $X^{*} \subseteq A$ of outcomes such that $\mathscr{C}^{*}=$ 
$\mathscr{C}\left(\tilde{s}_{C}, s[k]_{-C}\right)=\mathscr{C}\left(\tilde{s}_{C}, s\left[k^{\prime}\right]_{-C}\right)$ and $X^{*}=X\left(\tilde{s}_{C}, s[k]_{-C}\right)=X\left(\tilde{s}_{C}, s\left[k^{\prime}\right]_{-C}\right)$ for all $1 \leq k, k^{\prime} \leq|A|$. Also observe that $C(c, d) \in \mathscr{C}^{*}$ implies $C(c, d) \subseteq C$ for all $c, d \in A$. Moreover, for each $1 \leq m \leq|A|$ such that $a_{m} \in X^{*}$ we can find a $1 \leq k \leq|A|$ such that

$$
g\left(\tilde{s}_{C}, s[k]_{-C}\right)=a_{m}
$$

by setting $k=m^{\prime}$, where $m^{\prime}$ satisfies

$$
m=1+\left(\left(m^{\prime}+\sum_{i \neq i^{*}} \tilde{s}_{i}^{3}\right) \bmod \left|X^{*}\right|\right) .
$$

As a consequence we may assume that $b \notin X^{*}$, for otherwise there were some $1 \leq k \leq|A|$ with $g\left(\tilde{s}_{C}, s[k]_{-C}\right)=b$, which would be at variance with $(*)$. This, in turn, implies that $X^{*} \neq A$. Accordingly, there are some $c, d \in A$ such that $C(c, d) \in \mathscr{C}^{*}$ and $C(c, d) \subseteq C$.

Observe that for all $(c, d) \in \mathscr{C}^{*}$ we have $d=b$. Assume $d \neq b$ and consider the weak order $P \in \mathscr{W}(A)$ with $x \sim_{P} y>_{P} d$ for all $x, y \in A \backslash\{d\}$. Clearly, $P \in C(c, d)$ and, hence $P \in C$. Hence, it is not the case that $u_{P}(a)>u_{P}(b)$, which, however, is at variance with $(*)$.

It now follows that $\left|\mathscr{C}^{*}\right|=1$. To appreciate this, consider arbitrary $c, c^{\prime}, d, d^{\prime} \in A$ such that both $C(c, d) \in \mathscr{C}^{*}$ and $C\left(c^{\prime}, d^{\prime}\right) \in \mathscr{C}^{*}$. Then, $c=c^{\prime}=b$ and consider the weak order $P \in \mathscr{W}(A)$ with $x \sim_{P} y>_{P} b$ for all $x, y \in A \backslash\{b\}$. As both $P \in C(c, d)$ and $P \in C\left(c^{\prime}, d^{\prime}\right), C(c, d)$ and $C\left(c^{\prime}, d^{\prime}\right)$ are not disjoint. Hence, $C(c, d)=C\left(c^{\prime}, d^{\prime}\right)$.

Thus, $\mathscr{C}^{*}=\{C(c, b)\}$ and $X^{*}=\psi(c, b)$ for some $c \in A$. To show that $c=a$, assume for a contradiction that $c \neq a$. Then, there is a weak order $P \in \mathscr{W}(A)$ with $a>_{P} c>_{P} b$. Observe that $P \in C(c, b)$ and, hence, $P \in C$. Moreover, as obviously $c \in \psi(c, b)$, i.e., $c \in X^{*}$, there is some $1 \leq k \leq|A|$ such that $g\left(\tilde{s}_{C}, s[k]_{-C}\right)=c$. Now, however, $u_{P}(a)>u_{P}\left(g\left(\tilde{s}_{C}, s[k]_{-C}\right)\right)$, contradicting (*). Therefore, $C(a, b) \in \mathscr{C}^{*}$ and, by construction, $a R b$ holds.

The following corollaries show how the edge-mapping property can be employed. The first two can also easily be obtained by other means, but are included for illustrative purposes. Corollary 3 is slightly more substantial.

Corollary 1. Let $\chi$ be a comprehension condition. Then, every asymmetric relation on a finite set $A$ is obtainable through $\alpha$-effectivity under $\chi$.

Proof. Define $\psi: A \times A \rightarrow 2^{A}$ such that $\psi(a, b)=\{a\}$ for all $(a, b) \in R$. Obviously, $\psi$ is an edge mapping. Asymmetry of $R$, moreover, guarantees that no two edges in $R$ are $\psi$-exclusive. Hence, $R$ satisfies the edge-mapping property trivially. Theorem 3 then yields the desired result.

Corollary 2. Let $\chi$ be a comprehension condition and $R$ an irreflexive relation on a finite set $A$ such that there is some $a^{*} \in A$ with $b R a^{*}$ for no $b \in A$. Then, $R$ is obtainable through $\alpha$-effectivity under $\chi$.

Proof. Define $\psi: A \times A \rightarrow 2^{A}$ so that $\psi(b, c)=\left\{a^{*}, b\right\}$ for all $b, c \in A$ with $b R c$. Consider arbitrary such $b, c \in A$. Then, obviously, $b \in \psi(b, c)$. By irreflexivity of $R$, we have $c \neq b$ and, since $a^{*}$ is undominated, also $c \neq a^{*}$. Hence, $c \notin \psi(b, c)$ and we may conclude that $\psi$ is an edge mapping for $R$. Now observe that $a^{*} \in \bigcap_{(b, c) \in R} \psi(b, c)$. Accordingly, the relation $R$ has the edge-mapping property and Theorem 3 yields the desired result. 
Corollary 3. Let $\chi$ be a comprehension condition and $A$ a finite set of at least two outcomes. Then, the maximal irreflexive relation $\{(a, b) \in A \times A: a \neq b\}$ on $A$ is not obtainable through $\alpha$-effectivity under $\chi$.

Proof. Assume for a contradiction that the maximal irreflexive relation satisfies the edge-mapping property and let $\psi$ be the witnessing edge mapping. We first prove by induction on $k$ that for all $0 \leq k \leq|A|-2$ there are distinct $c_{1}, \ldots, c_{k} \in A$ such that the edges $(a, b),(b, a)$, $\left(a, c_{1}\right), \ldots,\left(a, c_{k}\right)$ are pairwise $\psi$-exclusive. For $k=0$ merely observe that $(a, b)$ and $(b, a)$ are $\psi$ exclusive as $a \in \psi(a, b)$ and $b \in \psi(b, a)$ by the definition of an edge mapping. For the induction step, assume that $a, b, c_{1}, \ldots, c_{k} \in A$ exist such that $(a, b),(b, a),\left(a, c_{1}\right), \ldots,\left(a, c_{k}\right)$ are pairwise $\psi$-exclusive. Having assumed the maximal irreflexive relation to satisfy the edge-mapping property in virtue of $\psi$, we have $\bigcap_{(x, y) \in X_{k}} \psi(x, y) \neq \emptyset$, where $X_{k}=\{(a, b),(b, a)\} \cup\left\{\left(a, c_{i}\right): 1 \leq i \leq k\right\}$. Observe, however, that $a \notin \psi(b, a), b \notin \psi(a, b)$ and $c_{i} \notin \psi\left(a, c_{i}\right)$ for each $1 \leq i \leq k$. Hence, there is some $c_{k+1}$ distinct from $a, b, c_{1}, \ldots, c_{k}$ such that $c_{k+1} \in \psi(a, b), c_{k+1} \in \psi(b, a)$ and $c_{k+1} \in \psi\left(a, c_{i}\right)$ for each $1 \leq i \leq k$. Now consider the edge $\left(a, c_{k+1}\right)$. Obviously, $a \in \psi\left(a, c_{k+1}\right)$. It follows that the edges $(a, b),(b, a),\left(a, c_{1}\right), \ldots,\left(a, c_{k+1}\right)$ are pairwise $\psi$-exclusive.

To conclude, consider the case where $k=|A|-2$. Then, $\left\{a, b, c_{1}, \ldots, c_{|A|-2}\right\}=A$ and the edges $(a, b),(b, a),\left(a, c_{1}\right), \ldots,\left(a, c_{|A|-2}\right)$ are pairwise $\psi$-exclusive. However, $a \notin \psi(b, a)$ and $x \notin \psi(a, x)$ for each $x \in A \backslash\{a\}$. Hence, $\bigcap_{(x, y) \in X_{|A|-2}} \psi(x, y)=\emptyset$, contradicting the assumption that the maximal irreflexive relation satisfies the edge-mapping property.

\section{Conclusion}

We characterized the structural restrictions of dominance relations in coalitional games that denote whether there is an effective coalition that unanimously prefers one outcome to another. We have shown that any irreflexive relation over a finite set can be obtained as the dominance relation of some ordinary, monotonic, and simple finite NTU game, even if we require that game to be induced by a non-cooperative game through $\beta$-effectivity. Dominance relations obtainable through $\alpha$-effectivity are characterized by a more restrictive condition, which we refer to as the edge-mapping property.

Many well-known dominance-based solution concepts from coalitional game theory (e.g., the core or stable sets) lack existence, uniqueness, or even both. Social choice theory, on the other hand, has produced solution concepts-e.g., the Banks set, the uncovered set, or the minimal covering set-of which existence, uniqueness, and several other desirable properties are guaranteed for asymmetric dominance relations on a finite set of alternatives (see, e.g., Laslier, 1997). An important question for future work is whether there are extensions of these concepts that retain most of their attractive properties for dominance relations that are not anti-symmetric.

\section{Acknowledgements}

We thank Felix Fischer and three anonymous referees for valuable comments. This material is based upon study supported by the Deutsche Forschungsgemeinschaft under grants BR 2312/31 and BR 2312/3-2. Preliminary results were presented at the fifth International Conference on 
Logic, Game Theory and Social Choice (Bilbao, June 2007), at the Dagstuhl Seminar on Computational Issues in Social Choice, and at the eighth Conference on Logic and the Foundations of Game and Decision Theory (Amsterdam, July 2008).

\section{Notes}

\footnotetext{
${ }^{1}$ There are also numerous concepts that take into account more structure of the social situation at hand. Thus, Fishburn (1977) distinguishes $C 1$ social choice functions, which merely involve the dominance relation, from $C 2$ and $C 3$ functions, for which this is not the case. In cooperative game theory, the dominance relation alone does not suffice to determine the bargaining set, the kernel, or the nucleolus. The Shapley value is defined on an entirely different basis.

${ }^{2}$ Although in the cooperative model of Bergin and Duggan (1999) utility is taken to be non-transferable, it is also crucially different from ours in that comprehensiveness is assumed throughout. Also the games they construct to prove their results involve an infinite number of strategies for the players. Consequently, without modification, Bergin and Duggan's Theorems 1 and 2 are not applicable to our finite model.

${ }^{3}$ This follows from Bergin and Duggan's characterization of NTU games supported by $\alpha$ - and $\beta$-effectivity in the comprehensive setting. However, if, for each coalition $C, V(C)$ is compact and convex, superadditivity is also satisfied for NTU games obtained through $\beta$-effectivity. The proof of this result is non-trivial and relies on Kakutani's fixed point theorem (Aumann, 1959; Aumann and Peleg, 1960). Also, in settings where comprehensiveness is assumed, this follows from Bergin and Duggan's Theorem 2.
}

\section{References}

Abdou, J. and Keiding, H. (1991), Effectivity Functions in Social Choice. Kluwer Academic Publishers.

Aumann, R. J. (1959), “Acceptable Points in General n-Person Games.” in: Tucker, A. W. and Luce, R. D. (eds.), Contributions to the Theory of Games IV, Volume 40 of Annals of Mathematics Studies, pp. 287-324. Princeton University Press.

(1961), “The Core of a Cooperative Game without Side Payments.” Transactions of the American Mathematical Society 98, 539-552.

Aumann, R. J. and Peleg, B. (1960), "Von Neumann-Morgenstern Solutions to Cooperative Games without Side Payments.” Bulletin of the American Society 66, 173-179.

Bergin, J. and Duggan, J. (1999), "An Implementation-Theoretic Approach to Non-cooperative Foundations." Journal of Economic Theory 86, 50-76.

Dutta, B. and Laslier, J.-F. (1999), "Comparison Functions and Choice Correspondences." Social Choice and Welfare 16 (4), 513-532.

Farquharson, R. (1969), Theory of Voting. Yale University Press.

Fishburn, P. C. (1977), "Condorcet Social Choice Functions." SIAM Journal on Applied Mathematics 33 (3), 469-489.

Hart, S. and MasColell, M. (1996), "Bargaining and Value." Econometrica 64 (2), 357-380. 
Kalai, E. and Samet, D. (1985), "Monotonic Solution Concepts to General Cooperative Games." Econometrica 53 (2), 307-328.

Kıbrıs, Ö. and Sertel, M. R. (2007), "Bargaining over a finite set of alternatives.” Social Choice and Welfare 28, 421-437.

Lahiri, S. (2007), "A Weak Bargaining Set for Contract Choice Problems." Research in Economics 61 (4), 185-190.

Laslier, J.-F. (1997), Tournament Solutions and Majority Voting. Springer-Verlag.

Luce, R. D. and Raiffa, H. (1957), Games and Decisions: Introduction and Critical Survey. John Wiley \& Sons Inc.

McGarvey, D. C. (1953), "A Theorem on the Construction of Voting Paradoxes." Econometrica 21 (4), 608-610.

Nash, J. (1950), “The Bargaining Problem.” Econometrica 18, 155-162. (1953), “Two-Person Cooperative Games.” Econometrica 21, 128-140.

Neumann, J. von and Morgenstern, O. (1947), Theory of Games and Economic Behavior. Princeton University Press, 2nd edition.

Rubinstein, A. (1982), “Perfect Equilibrium in a Bargaining Model.” Econometrica 50 (1), 97 109.

Schwartz, T. (1990), “Cyclic Tournaments and Cooperative Majority Voting: A Solution.” Social Choice and Welfare 7, 19-29.

Serrano, R. (1997), "A Comment on the Nash Program and the Theory of Implementation." Economic Letters 55, 203-208.

Taylor, A. D. and Zwicker, W. S. (1999), Simple Games. Desirability Relations, Trading, Pseudoweightings. Princeton University Press.

\section{Authors}

Felix Brandt

Institut für Informatik

Ludwig-Maximilians-Universität München

Oettingenstraße 67

80538 Munich

Germany

telephone: +49-89-21809691

fax: +49-89-2180-9338

email: felix.brandt@tcs.ifi.lmu.de 
Paul Harrenstein

Institut für Informatik

Ludwig-Maximilians-Universität München

Oettingenstraße 67

80538 Munich

Germany

telephone: +49-89-2180-9405

fax: +49-89-2180-9338

email: paul.harrenstein@tcs.ifi.lmu.de 